\title{
LE CHÊNE VERT EN RÉGION MÉDITERRANÉENNE
}

M. BARBERO - R. LOISEL

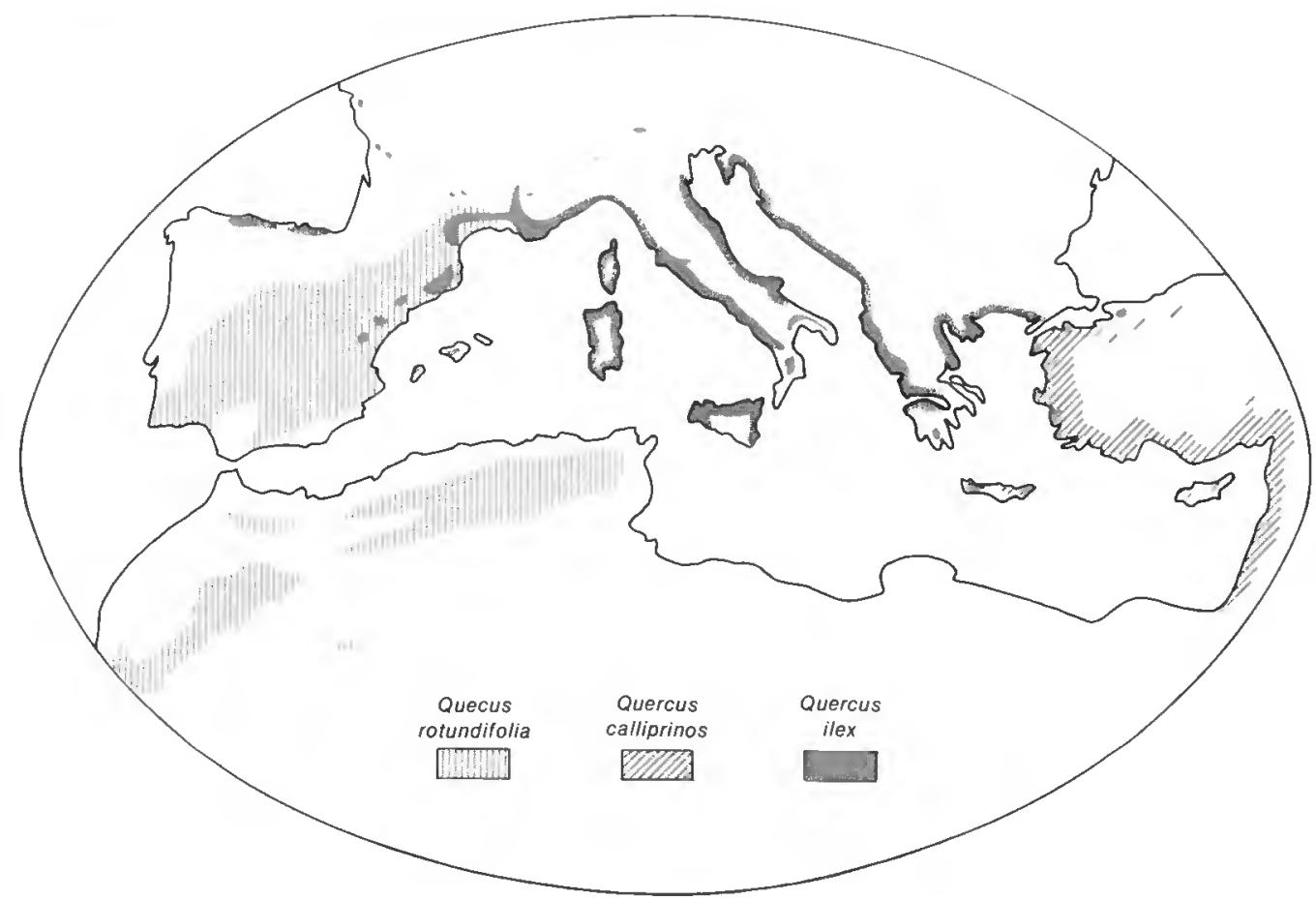

RÉPARTITION GÉOGRAPHIQUE

L'aire du Chêne vert s'étend sur l'ensemble du bassin méditerranéen ; c'est ce que traduisent les flores en qualifiant l'espèce de circumméditerranéenne.

Cependant, ceux qui ont prospecté tous les pays méditerranéens savent qu'en termes d'occupation du tapis végétal, le Chêne vert joue un rôle indiscutablement plus important dans la partie occidentale du bassin méditerranéen que dans sa partie orientale.

La chaîne de l'Apennin constitue un excellent exemple permettant d'apprécier le phènomène: si, sur son versant méditerranéen, les chênaies vertes remontent largement sur les reliefs, sur son versant adriatique, par contre, elles se cantonnent le plus souvent aux altitudes inférieures, en un liseré littoral et sublittoral. 


\section{BARBERO - R. LOISEL}

II en est de même sur le littoral des Balkans et ce jusqu'en Grèce. Dans le Péloponnèse (Barbero et Quezel, 1976), le Chêne vert est absent des zones côtières où il est remplacé notamment par Quercus coccifera et Quercus pseudococcifera; on ne le rencontre que dans des situations écologiques particulières comme les gorges.

En Grèce nord-occidentale et en Turquie d'Europe, s'observe une répartition identique à celle constatée sur le littoral des Balkans.

Par contre, en Anatolie, le Chêne vert devient d'une très grande rareté puisqu'il n'apparaît qu'en quelques points - région d'Istanbul, littoral de la Mer Noire à Samsun et Massif du Samsoun dâg, près de Kusâdasi.

Le Chêne vert a également colonisé les îles méditerranéennes: Baléares $(O$. de Bolós et René Molinier, 1958, O. de Bolós, R. Molinier et P. Montserrat, 1970), Corse, Sardaigne et Sicile où il joue un rôle important. On le retrouve également en Crète où il est cependant rare (Barbero et Quezel, 1979).

Le Chêne vert, enfin, s'observe hors de la région méditerranéenne. C'est le cas en Espagne, dans les zones Jittorales et juxtalittorales de la région Cantabrique, en France avec les avant-postes de la bordure sud-occidentale du Massif Central (Vernet, 1972) et du littoral de Vendée, en Italie sur le revers des Alpes (Barbero, 1979).

\section{TAXONOMIE ET ÉCOLOGIE}

De nombreux travaux ont été consacrés à la taxonomie du Chêne vert; parmi les plus anciens, nous citerons ceux d'Albert (in Albert et Jahandiez, 1908) qui ne distingue pas moins de 31 variétés d'Yeuse, et parmi les plus récents, ceux de C. Saenz de Rivas $(1969,1970,1972)$. A la suite de ces auteurs, on admet souvent au sein du groupe Chêne vert, deux espèces différentes - Quercus ilex et Quercus rotundifolia - mais il serait également possible de considérer qu'il s'agit de deux variétés.

Quercus rotundifolia occuperait l'ensemble de l'Afrique du Nord et une bonne partie de l'Espagne méditerranéenne. Au contraire Quercus ilex n'apparaîtrait vers le nord qu'à partir de Valence. Ce serait l'essence dominante du midi méditerranéen français dans le groupe des Chênes sclérophylles.

Au-delà des caractères morphologiques analysés par les auteurs qui permettent de distinguer Quercus ilex et Quercus rotundifolia et notamment le nombre de nervures des feuilles -6 à 7 chez Quercus rotundifolia, 8 à 9 et plus chez Quercus ilex - les particularités écologiques des deux essences jouent aussi un rôle important. Les aspects morphologiques - port droit de Quercus ilex, port buissonnant de Quercus rotundifolia - ne nous semblent pas, par contre, devoir être retenus, le traitement sylvicole étant en effet souvent déterminant dans la variabilité phénotypique des individus. Ii existe d'ailleurs en Afrique du Nord des Chênes de belle forme non buissonnants se rapportant à Quercus rotundifolia.

Par contre, les données écologiques et en particulier bioclimatiques méritent qu'on insiste davantage. Ainsi en Espagne Rivas-Martinez (1975) a montré que Quercus ilex colonisait les régions tempérées aussi bien en zone littorale et sublittorale, qu'en montagne humide. Aux Baléares, le caractère océanique du climat favorise aussi son développement. Par contre Quercus rotundifolia occupe les stations à climat continental sec et à fréquentes gelées tardives. 


\section{Biologie et forêt}

Cependant si ces conclusions s'appliquent à l'Espagne, elles doivent être corrigées pour l'ensem. ble de l'Afrique du Nord où le Chêne occupe les zones bioclimatiques se succédant entre le semiaride et l'humide, voire localement le perhumide comme en certains points du Rif.

Compte tenu de ces observations régionales nous nous sommes appliqués à étudier les populations de Chêne vert s.l. partout où l'occasion nous en était donnée.

C'est ainsi que depuis la Ligurie jusqu'en Turquie pontique (Quezel, Barbero et Akman, 1979) nous avons toujours rencontré des peuplements dont les individus à longues feuilles montrent un grand nombre de nervures. En outre, tous les groupements rencontrés là occupent la variante fraîche du bioclimat méditerranéen humide et à un moindre degré subhumide. Se rapportent aussi à ce groupe les chênaies vertes de Vendée et du versant italien des Alpes (Mondino, 1966; Barbero, 1979, Ozenda, 1966).

Pour ce qui est de la Provence et du Languedoc, il semble que l'on se trouve en présence de populations montrant une grande variabilité locale. Dans certaines dominent Quercus rotundifolia, notamment sur les crêtes et les vires, tandis que d'autres, dans les stations plus humides, sont représentées en grande partie par Quercus ilex. Enfin il faut signaler de très nombreux groupements où sont manifestement en mélange tous les intermédiaires entre Quercus rotundifolia et Quercus ilex.

Si l'on considère à présent les exigences géopédologiques de ces deux essences sur l'ensemble de leur aire de répartition, on doit conclure à une très large plasticité édaphique. Les caractères chimiques en particulier ne sont pas un obstacle à leur répartition. Les deux chênes en outre se rencontrent aussi bien sur les sols superficiels que profonds oú ils sont alors, là où la pression humaine s'atténue, en passe d'être sérieusement concurrencés par les feuillus caducifoliés.

Ce sont donc essentiellement les caractères climatiques qui jouent un rôle essentiel dans la localisation des deux essences.

\section{SITUATION ALTITUDINALE}

En ce qui concerne, enfin, la répartition altitudinale du Chène vert s.l., sur l'ensemble de son aire, on est conduit à une double constatation suivant que l'on se réfère aux données de la latitude ou à celle de la longitude.

II est facile de mettre en évidence une nette variation des limites altitudinales supérieures de la répartition du Chêne vert en fonction de la latitude. En Vendée, il occupe les zones côtières et ne dépasse pas 200-300 m au lac de Garde. II atteint 500-600 m dans la Drôme, $1000 \mathrm{~m}$ sur l'adret du Lubéron; en Basse-Provence il est très fréquent et très abondant depuis le littoral jusqu'à 800 $900 \mathrm{~m}$ en moyenne, voire $1200 \mathrm{~m}$ localement, dans les zones thermiques les plus favorables. Dans les parties les plus méridionales de son aire et notamment sur le Grand Atlas, le Chêne vert peut organiser des peuplements continus jusqu'à 2 400-2 $500 \mathrm{~m}$ d'altitude.

On peut également mettre en évidence une franche variation altitudinale en fonction de la longitude. Ainsi, pour une même latitude, le Chêne vert atteint 1200 à $1300 \mathrm{~m}$ dans la région de Valen. cia alors que ses peuplements continus se limitent à $700 \mathrm{~m}$ au Sansum dâg en Turquie. Il en est de même pour les colonies de Sansoun, sur le littoral de la Mer Noire, et les Massifs pyrénéens orien. taux où, bien que la latitude soit identique, le Chêne vert atteint respectivement 700 et 1100 . $1200 \mathrm{~m}$. 
La position latitudinale permet donc d'expliquer le décalage altitudinal qui existe entre les peuplements de Chêne vert dans la partie occidentale du bassin méditerranéen, mais ne convient plus lorsqu'on compare entre elles les régions occidentale et orientale.

Ozenda (1975) a pu évaluer ce décalage altitudinal en ce qui concerne les peuplements mésoméditerranéens (cf. infra) du bassin méditerranéen occidental. Ils se localisent en moyenne entre 400 et $800 \mathrm{~m}$ dans les Alpes-Maritimes (Ozenda, 1966) et entre 600 et $1200 \mathrm{~m}$ dans le Haut Atlas. Des conclusions identiques ont été obtenues quant aux chênaies mésoméditerranéennes du Grand Atlas (Barbero, Quezel, Rivas-Martinez, 1979) mais il est évident que dans ce cas précis, le Chêne vert constitue aussi de beaux peuplements appartenant à d'autres structures de végétation, au-dessus de l'étage mésoméditerranéen.

En région méditerranéenne les classifications les plus récentes sont celles d'Ozenda (1975) et de Quezel (1974) qui diffèrent assez sensiblement pour les zones culminales.

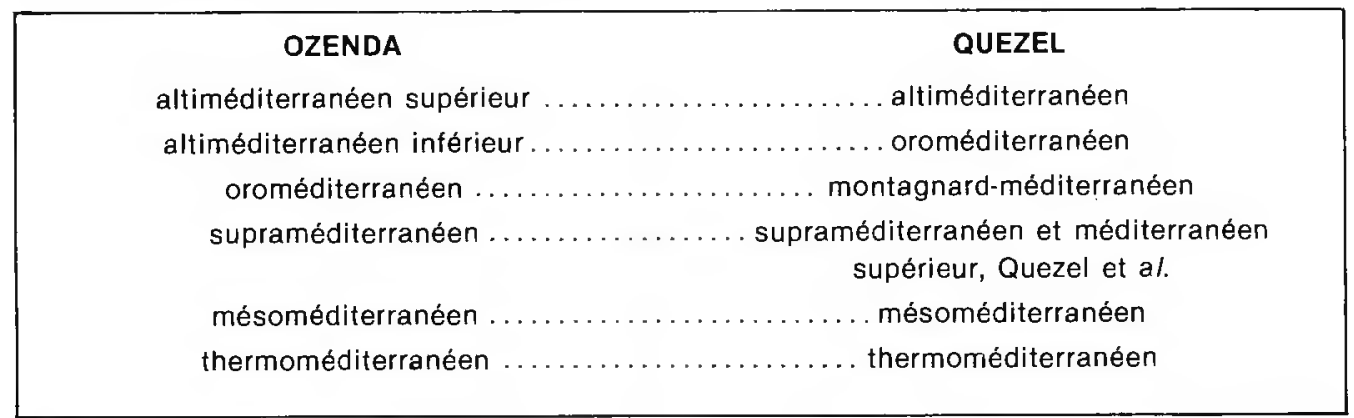

Ces deux points de vue conduisent à une même caractérisation de l'étage mésoméditerranéen. Cependant un décalage important apparaît à partir de l'oroméditerranéen puisque cet étage identifié par Ozenda est décalé en altitude au-dessus du montagnard-móditerranéen dans la conception de P. Quezel.

Une correspondance entre ce système de classification et celui des montagnes européennes est intéressante à envisager. Les corrélations se présentent comme suit :

\begin{tabular}{|c|c|}
\hline $\begin{array}{c}\text { Montagnes méditerranéennes } \\
\text { altiméditerranéen ..... } \\
\text { oroméditerranéen ..... } \\
\text { montagnard-méditerranéen.. } \\
\text { supraméditerranéen et } \\
\text { méditerranéen supérieur. . } \\
\text { mésoméditerranéen } \\
\text { thermoméditerranéen }\end{array}$ & $\begin{array}{l}\text { Montagnes européennes } \\
\ldots \ldots \ldots \text { alpin } \\
\ldots \ldots \text { subalpin } \\
\ldots \ldots \text { montagnard } \\
\ldots \ldots \ldots \text { collinéen }\end{array}$ \\
\hline
\end{tabular}

\section{ASPECTS FLORISTIQUES ET DYNAMIQUES DES CHÊNAIES VERTES}

La chênaie verte mésoméditerranéenne correspond à une unité floristique bien reconnaissable d'un bout à l'autre du bassin méditerranéen. En son sein, en effet, les arbustes et les lianes à feuilles coriaces sont importants : Viburnum tinus, Phillyrea media, Smilax aspera, Arbutus unedo, Lonicera implexa, Juniperus oxycedrus ssp. oxycedrus. 


\section{Biologle et forêt}

Mais ces espèces peuvent organiser, dans leurs mélanges, des structures de végétation différentes :

- chênaies ouvertes à Pistacia terebinthus, Juniperus oxycedrus aux altitudes inférieures, à Buxus sempervirens, Juniperus communis aux altitudes supérieures;

- chênaies à couronnes jointives à Rosa sempervirens, Phillyrea media, Lonicera etrusca, Hedera helix.

Si les premières structures correspondent à des milieux ouverts probablement liés à l'influence de l'homme et des troupeaux et si d'autres apparaissent encore lorsque l'évolution régressive de l'écosystème Chênaie est engagée, par contre les chẻnaies à couronnes jointives représentent bien, malgré leur extrême rareté, des forêts d'équilibre proches du climax.

Toutes ces chènaies qui s'encartent dans l'alliance Quercion ilicis ont été étudiées en détails sur la Péninsule ibérique (O. de Bolós, 1962 ; Rivas-Martinez, 1975 ; Rivas-Goday et Rivas-Martinez, 1979), en France méditerranéenne (Molinier, 1934 ; Loisel, 1971, 1976...), en Italie (Gentile, 1969), dans les Balkans septentrionaux (1976), en Grèce (Barbero et Quezel, 1976). Elles sont caractérisées par: Carex distachya, Viola denhardtii, Epipactis microphylla, Oryzopsis paradoxa, Carex olbiensis, Lonicera etrusca, Rosa sempervirens.

En Crète déjà, l'apparition d'un cortège floristique particulier les placent dans une alliance spéciale : Cyclamini-Quercion (Barbero et Quezel, 1979) définie par : Cyclamen creticum, Aristolochia cretica, Chamaecytisus creticus, etc.

Au sein du complexe chênaies vertes, certaines associations ont une grande valeur biogéographique ; par exemple:

Le Quercetum ilicis galloprovinciale [ou Viburno-Quercetum (Rivas-Martinez, 1975)] qui s'étend de la Catalogne à la Provence, occupe pour l'essentiel la variante fraíche de la zone bioclimatique subhumide. Au contraire la variante fraiche de la zone bioclimatique humide est occupée, en partie, entre la Ligurie et les Balkans, par l'Orno-Quercetum ilicis. Enfin, en Grèce littorale et sublittorale, en Turquie d'Europe et d'Asie, le Chêne vert domine surtout dans la variante tempérée de la zone subhumide avec l'association Andrachno-Quercetum ilicis.

Précisons enfin que d'un bout à l'autre de la Méditerranée, nous avons été frappés par la grande stabilité floristique des chênaies mixtes sclérophylles à Laurus nobilis qui constitueraient des forêts mésophiles et thermophiles en raison de leur présence sur les sols profonds à nappe proche de la surface et dans les zones littorales.

Toutes ces forêts mésoméditerranéennes ont pour caractéristiques d'évoluer vers des groupements climaciques où participent de nombreuses espèces sylvatiques : Carex distachya, Viola dehnhardtii, Epipactis microphylla, Oryzopsis paradoxa, Carex olbiensis. Par dégradation elles passent progressivement vers des forêts-garrigues très ouvertes où prospèrent les caractéristiques des manteaux forestiers qui s'encartent dans les Pistacio-Rhamnetalia : Quercus coccifera, Osyris alba, Osyris quadridentata, Coronilla juncea, Jasminum fruticans, Euphorbia dendroides, etc.

Pour la zone à Quercus rotundifolia de l'Espagne et du Maroc, d'autres unités phytosociologiques ayant valeur d'alliance ont été définies.

Ainsi en Espagne cet ensemble regroupe les chênaies vertes soumises à un climat plus continental. Les lianes et les arbustes sont sensiblement moins fréquents que dans les forêts mésoméditerranéennes typiques.

Au Maroc, par contre, du fait de l'amplitude altitudinale des chênaies vertes, deux alliances et plusieurs associations ont pu être définies. Le Querco-Oleion à l'étage thermoméditerranéen est 


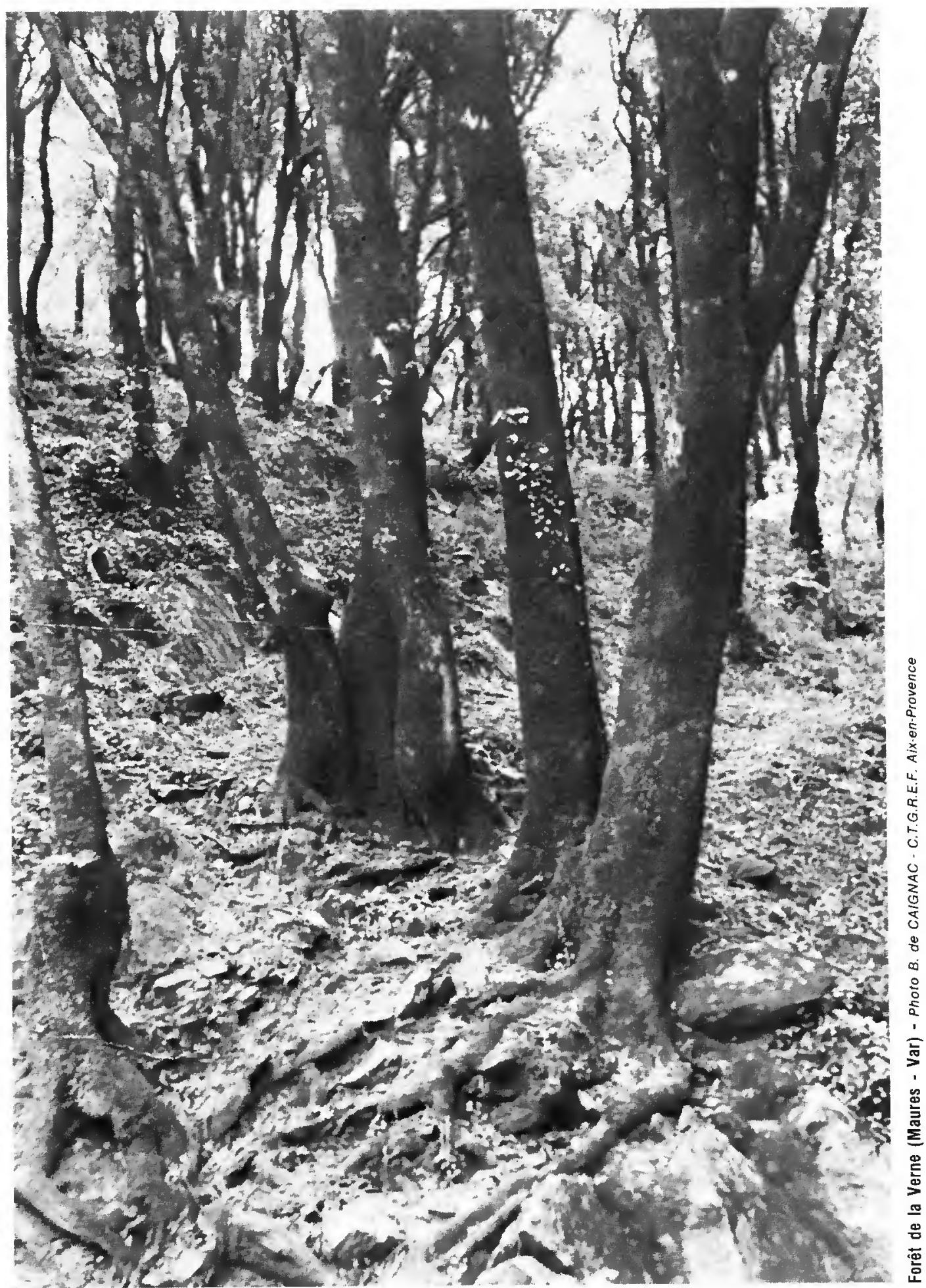


représenté entre autres par une chênaie thermophile décrite sous le vocable de Smilaci-Quercetum rotundifolia. Ses caractéristiques sont: Arisarum vulgare var. subexsertum, Gennaria diphylla, Olea europaea var. silvestris Ruscus hypophyllum, Rubia peregrina var. longifolia, Smilax aspera ssp. mauretanica.

II sera intéressant de préciser la répartition de tels types de yeuseraies jusqu'ici peu étudiés sur le pourtour du Bassin méditerranéen.

De l'étage mésoméditerranéen à l'étage montagnard-méditerranéen, certains massifs du Maroc sont couverts par des associations forestières dans lesquelles le Chêne vert est l'essence de base dominante. L'étage des feuillus caducifoliés correspondant au supraméditerranéen est alors remplacé par un étage de sclérophylles pour lequel le nom d'étage méditerranéen supérieur a été proposé.

L'alliance phytosociologique dans laquelle s'intègrent toutes ces forêts est le Ba/ansaeoQuercion. Celles-ci atteignent $1600 \mathrm{~m}$ sur le Rif, $1800 \mathrm{~m}$ sur le Moyen Atlas, $2000 \mathrm{~m}$ sur le Haut Atlas. Elles sont caractérisées par Anarrhinum pedatum, Balansaea glaberrima, Bupleurum montanum, Genista jahandiezi, Luzula atlantica, Narcissus watieri, Ptilostemon dyricola, Origanum elongatum.

Les structures suivantes peuvent être distinguées :

- une chênaie à Phillyrea media, Arbutus unedo, Lonicera implexa, entre 700 et $1200 \mathrm{~m}$, correspondant au mésoméditerranéen typique,

- une chênaie à Galium ellipticum, Paeonia div. sp., Moehringia trinervia, Aristolochia baetica, Festuca triflora au-dessus de $1200 \mathrm{~m}$ et représentant le méditerranéen supérieur dans les variantes froides des zones subhumide et plus rarement humide,

- au-dessus de $1600 \mathrm{~m}$ en moyenne, dans les variantes froide et très froide de la zone subhumide, le Chêne vert caractérise l'étage montagnard.

II constitue souvent des peuplements mlxtes avec le Cèdre, généralement sur les adrets et dans les zones de fortes pentes. C'est le cas localement sur le Rif et plus généralement sur le Moyen Atlas et le Grand Atlas oriental.

Par contre, dans le Grand Atlas occidental et central quand les conditions deviennent plus xériques et plus froides, le Chêne vert élimine complètement le Cèdre. On se trouve souvent alors en présence de chênaies où sont absentes ou rarissimes les caractéristiques sylvatiques du Balansaeo-Quercion. II est vrai aussi que l'on entre dans une zone bioclimatique différente : la zone semi-aride dans ses variantes froide à très fraîche.

En zone semi-aride d'ailleurs, le Chêne vert intervient à titre d'essence associée ou d'essence dominante. Mais les forêts qu'il constitue alors sont qualifiées de présteppiques en ce sens qu'elles n'offrent pratiquement plus d'espèces sylvatiques.

On retrouve dans ces structures, des espèces des Pistacio-Rhamnetalia mais aussi des caractéristiques d'une unité nouvelle : les Ephedro-Juniperetalia qui occupent dans les parties internes du Haut Atlas les variantes froides de la zone bioclimatique semi-aride.

Ainsi par exemple:

- l'étage mésoméditerranéen présteppique est représenté jusqu'à $1400 \mathrm{~m}$ et en partie par des groupements mixtes de Quercus rotundifolia et de Genévrier rouge ou des groupements purs de Genévrier dont l'étude est actuellement en cours et dans lesquels Ephedra major joue localement un rôle important ; 


\section{BARBERO - R. LOISEL}

- l'étage méditerranéen supérieur présteppique est constitué, entre 1400 et $1800-1900 \mathrm{~m}$, en zone semi-aride (variante froide et pluviométrie ne dépassant pas $500 \mathrm{~mm}$ ) par des forêts mixtes de Quercus rotundifolia, Buxus balearica, Juniperus oxycedrus ssp. rufescens ;

- l'étage montagnard présteppique, au-dessus de $1900 \mathrm{~m}$ et jusqu'à $2200-2300 \mathrm{~m}$, offre dans les massifs les plus internes à bioclimat semi-aride très froid, des groupements mélangés de Chêne vert et de Genévrier thurifère. C'est le cas notamment dans les parties orientale et centrale du Haut Atlas et sur le Moyen Atlas oriental. Dans les parties plus océaniques, l'étage montagnard présteppique n'a plus de Genévrier thurifère. Le Chêne vert est la seule essence de la torêt.

Dans cet étage s'infiltrent déjà de nombreuses espèces des garrigues à xérophytes épineux (Quézel, 1957): Cytisus balansae, Ptilotrichum spinosum, Bupleurum spinosum, Astragalus ibrahinianus, Ormenis scariosa.

Entre 2300 et $2600.2700 \mathrm{~m}$ la Juniperaie à Juniperus thurifera dominant apparaît. Le Chêne vert n'existe alors qu'en individus isolés dans la Juniperaie climatique (Lecompte, 1969).

Ainsi c'est vraiment à deux entités trẻs diftérentes qu'appartiennent les chênaies vertes de I'Atlas:

- une entité avec des espèces sylvatiques bien caractérisées occupant tous les étages de végétation depuis le thermoméditerranéen supérieur jusqu'au montagnard méditerranéen et s'intègrant aux Quercetalia ilicis dans les zones bioclimatiques subhumide et à un moindre degré humide,

- une entité où les espèces sylvatiques ont disparu et où se développent des forêts présteppiques s'intégrant soit aux Pistacio-Rhamnetalia, soit aux Ephedro-Juniperetalia et représentant les variantes tempérées à trés froides de la zone semi-aride.

II existe donc des différences trés importantes dans la répartition des essences du groupe Chêne vert entre le bassin occidental et le bassin oriental de la Méditerranée.

Dans le bassin occidental, le Chêne vert occupe, quant à sa répartition altitudinale, une palette remarquable d'étages, surtout en Afrique du Nord. Cette répartition tient au fait que s'y manifestent les différentes variantes des bioclimats subhumide et semi-aride.

Au contraire, en Méditerranée orientale, le Chêne vert reste strictement cantonné à l'étage mésoméditerranéen et encore en son sein il y est fortement concurrencé par Quercus coccifera et Quercus pseudococcifera dans le Péloponnèse et en Crète ainsi que par Quercus calliprinos en Turquie, Syrie, Liban... Ce chêne représente même localement l'étage méditerranéen supérieur. Dès qu'on s'élève en altitude dans le bassin méditerranéen oriental et même dès l'étage supraméditerranéen, les conifères (Pinus nigra, localement Pinus brutia, Abies cephalonica, Abies borisii-regi, Cedrus libani) et les feuillus caducifoliés de type Quercus et localement Fagus jouent un rôle déterminant dans le tapis végétal, ce qui traduit, malgré une sécheresse plus prolongée qu'en Méditerranée occidentale, de bonnes potentialités forestières.

En Méditerranée occidentale il ne faudrait pas pour autant négliger le rôle des feuillus caducifoliés, nofamment en zone bioclimatique humide.

Ainsi dans le Midi méditerranéen français beaucoup d'auteurs (Ozenda 1966, Loisel, 1976, Thinon, 1979, ...) malgré les positions opposées par les tenants de l'école zuricho-montpellieraine, ont insisté sur le rôle déterminant du Chêne pubescent à l'étage méditerranéen, sur les remarquables potentialités actuelles de cette essence, en remontée biologique, et sur la nécessité de réduire trés sensiblement l'aire actuelle du Chêne vert qui aurait été favorisée, en de nombreux points, par les dégradations. La chénaie pubescente primitive, une fois éliminée (Pons et Vernet, 1971), a été progressivement remplacée par le Chêne vert qul supportait beaucoup mieux l'exploitation, en rai- 


\section{Biologie et forêt}

son de sa faculté remarquable à régénérer de souche et à se prêter à un meilleur traitement en taillis. Les dégradations massives de la chênaie pubescente auraient en outre provoqué une remontée de xéricité très favorable au Chêne vert qui aurait été ainsi favorisé en de nombreux points aux dépens du Chêne pubescent.

Des conclusions identiques sont applicables à l'Espagne méditerranéenne où en certaines régions - sud-ouest de la Péninsule - sur substrats siliceux, $Q$. faginea envahit les suberaies, tandis que sur calcaire, certaines chènaies sur sols profonds à Quercus rotundifolia subissent le même processus. Il en est de même dans la région de Cadiz oủ certaines chênaies sclérophylles représenteraient, en ambiance méditerranéenne tempérée et pluvieuse, des territoires potentiels pour la forêt méditerranéenne de Quercus canariensis bien développée par ailleurs.

Nous avons observé le même phénomène dans le Rif littoral occidental, à l'étage thermoméditerranéen. A l'étage mésoméditerranéen, certaines chênaies de Quercus rotundifolia du Rif montrent aux expositions nord, une forte infiltration de Quercus faginea ssp. maroccana et sur silice de Quercus pyrenaica, voire Quercus canariensis.

En Algérie et en Tunisie, des phénomènes identiques s'observent. En de nombreux points de Kroumirie et de Kabylie, la suberaie sclèrophylle est nettement infiltrée de Quercus mirbeckii. Sur la dorsale tunisienne (Djebel Serdj), comme sur l'Atlas Tellien algérien, on assiste à une recolonisation locale de certains taillis de Quercus rotundifolia par le Chène-liège et même localement (sur l'Atlas Tellien notamment) par le Chêne zéen et divers feuillus caducifoliés comme Acer obtusatum, Merisier, etc. Cette recolonisation semble liée à la migration de populations qui tendent à quitter ces zones montagneuses pour gagner les villes.

II est donc indéniable qu'en zones bioclimatiques humide et subhumide, le Chêne vert occupe, en de très nombreux points, des territoires qui reviendraient, sans trop de difficultés, aux caducifoliés, pour peu que la pression humaine sur la végétation naturelle s'allége. On peut donc concevoir que les zones actuellement occupées par le Chêne vert représentent :

- soit des territoires de chênaies vertes climaciques,

- soit des territoires de forêts mixtes : feuillus et sclérophylles,

- soit des territoires de chênaies caducifoliées.

Des donnėes historiques, de plus en plus nombreuses, viennent appuyer ces observations actuelles.

Qu'elles portent sur du matériel pollinique ou sur des charbons de bois récoltés dans les sédiments, elles apportent de précieuses précisions sur le passé de la végétation et notamment sur le rôle joué par le Chêne vert et les variations de son importance au niveau du tapis végétal. Parmi ces études il convient de citer celles de Pons, 1964, 1969, Pons et Vernet, 1971, Pons et al., 1975, Vernet, 1972, Bernard, 1971, 1972, Triat-Laval, 1978, Thinon, 1979.

Ces travaux nous apprennent notamment :

- le Chêne vert est connu depuis l'Oligocène mais ce n'est qu'au cours du Villafranchien qu'il est soumis à des conditions climatiques (xéricité et froid) comparables à celles qu'il rencontre actuellement en certaines régions d'Afrique du Nord,

- l'antagonisme, pour l'occupation du terrain, entre le Chêne vert et le Chène pubescent, en région méditerranéenne française, est ancien et souvent à l'avantage du second.

Ce n'est qu'avec la sédentarisation des populations humaines et leurs actions sur la végétation (déboisements) que le Chêne vert montre une grande extension relativement récente puisqu'elle est postérieure à l'Atlantique moyen. 


\section{BARBERO - R. LOISEL}

\section{UTILISATION DE LA CHENAIE VERTE}

Les problèmes de l'utilisation de la chênaie verte se posent nécessairement dans la perspective d'un aménagement bien défini tenant compte des caractéristiques écologiques et biogéographiques qui viennent d'être développées et qui conduisent à la différenciation d'un nombre important de chênaies et par conséquent de structures forestières.

On ne saurait donc, en termes d'utilisation rationnelle de l'espèce, traiter de la même manière les yeuseraies dans les différentes zones bioclimatiques. En particulier des garde-fous sérieux doivent être mis en place notamment dans toute la zone semi-aride où la production du Chène vert est très faible. On a pu montrer que, dans cette zone, les meilleures placettes du Moyen Atlas avaient 73 stères par hectare et celles du Grand Atlas n'atteignaient que 64 stères par hectare.

Le seul aménagement possible de ce type de forêts semi-arides ou présteppiques est encore le régime des taillis malgré tous les inconvénients qu'on lui connaît et les abus qu'il a permis (rotation de 18 à 20 ans qui n'a pas été respectée dans certains cas alors qu'il conviendrait d'allonger ces délais). Cependant le régime des taillis permet aux espèces pastorales d'occuper les zones de lumière entre les cépées. L'aménagement sylvo-pastoral, avec introduction d'espèces fourragères associées à une mise en défend par rotation, serait alors la meilleure façon d'utiliser l'yeuseraie. Dans une telle perspective, étant données les conditions écologiques très sévères, une limitation stricte de la charge et le contrôle régulier de ses effets sur le milieu s'imposent.

Dans les zones bioclimatiques subhumide et humide, les données que nous avons pu établir pour le Maroc dans le cadre de la R.C.P. 427 montrent des productions nettement supérieures aux précédentes.

Ainsi, par exemple, à lfrane, le volume moyen est de 162 stères par hectare, à Azrou, il est de 130 stères par hectare, à Ain Leuh de 135 stères par hectare. Ces trois stations se situent la première en zone humide et les deux autres en zone subhumide fraîche. On peut donc penser que dans de telles forêts, à conditions que la pression actuelle du troupeau soit rigoureusement contrôlée, le traitement en futaie sur souche est possible.

D'ailleurs les futaies d'Ifrane sont fort remarquables et certainement parmi les plus belles du bassin méditerranéen. Elles donnent une excellente idée de ce que peut être une forêt bien gérée de Chêne vert. Le seul problème qui se pose, au regard d'une utilisation économique éventuelle de la Chênaie verte, est certainement celui de la qualité du bois de I'Yeuse. Les études technologiques ont montré qu'il s'agit d'un bois lourd, dur, à fort retrait et à caractéristiques mécaniques moyennes. Son séchage est long et délicat. De plus les défauts du bois lui-même sont nombreux et de ce fait il est très difficile d'obtenir des pièces de dimension importante. II faudra donc, en fonction de l'utilisation, sélectionner les grumes.

Néanmoins, outre l'emploi local qui a été fait du Chêne vert - menuiserie de bâtiment, charpentes grossières, ébénisterie sur les plus belles pièces - on pourrait envisager son utilisation dans l'industrie des panneaux de fibres et de particules. Mais il est évident que l'on est loin de la qualité des Chênes caducifoliés, pour l'ébénisterie notamment.

C'est pourquoi au Maroc, comme ailleurs, en raison de la faible valeur économique du bois de Chêne vert, la plupart des chênaies ont été traitées en taillis pour le bois de chauffe ou pour la production de charbon de bois.

Le processus de carbonisation qui était fréquent en région européenne est aujourd'hui quasiment stoppé alors qu'il est encore actif en Afrique du Nord où la chênaie verte paie un lourd tribut à ce mode d'exploitation. 


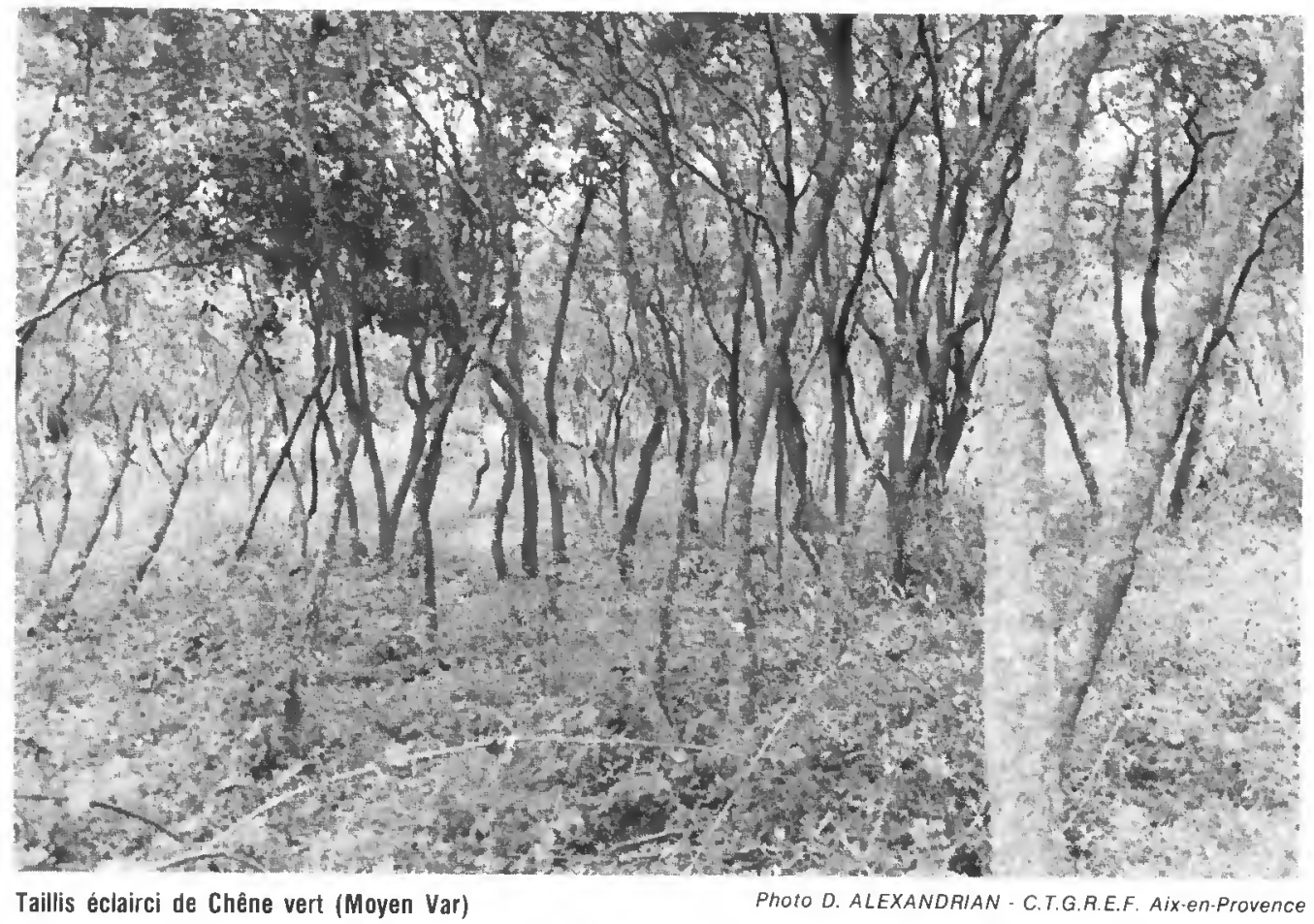

En France méditerranéenne, et dans le Var en particulier où nous avons pu obtenir des informations sur l'utilisation de la chênaie, il a pu être établi que le taillis était utilisé pour le bois de chauffe et la carbonisation avec une rotation de 18 ans.

Quelques années après la coupe, les recépées constituaient des milieux ouverts riches en espèces fourragères, qui étaient alors parcourus par les troupeaux d'ovins. Ce n'est que lorsque le taillis se fermait qu'il était inutilisé par l'animal.

Des futaies cependant ont pu demeurer en place dans des secteurs très précis en raison d'une protection liée à la propriété. Dans le Var, on peut citer à ce titre les magnifiques yeuseraies de la Chartreuse de la Verne.

Quelques données de productivité en bois ont été fournies par Lossaint et Rapp (1978) pour la forêt de Rouquet au nord-ouest de Montpellier : la production annuelle moyenne épigée est de $1,7 \mathrm{t} / \mathrm{ha}$ de bois, 0,9 t/ha de pousses et 3,9 t/ha de litières.

Enfin, les territoires potentiels de la chênaie étaient souvent soumis à des pratiques culturales; les céréales étaient notamment utilisées. Quelques gros arbres, sous lesquels on cultivait, étaient laissés en place. Le blé était semé sur les bonnes terres de bas-fonds, territoire d'élection de la chênaie pubescente méditerranéenne, l'avoine et l'orge étaient plantés en complément entre les chênes verts. Leur récolte était plus précoce; celle-ci réalisée, le troupeau passait puis on pratiquait le brûlis. Certaines années de déficit fourrager, les arbres étaient émondés et leur feuillage donné aux bêtes.

Un autre mode d'utilisation était la forêt parcours permanent. Des arbres épars étaient laissés en place et entre eux, les buissons et arbustes éradiqués; le tapis herbacé pouvait alors se développer sans entrave. Les grands chênes témoins produisaient périodiquement des glandées importantes utilisées par les animaux. 
Les vestiges de tels modes d'occupation de la forêt se retrouvent encore dans certaines régions du Var. De grands chẻnes verts mitant irrégulièrement le taillis de colonisation récente sont les témoins vivants de ces pratiques ancestrales.

De même, le taillis de Chêne vert a aujourd'hui repris sur d'anciens territoires d'olivettes. II n'est pas rare, en effet, sur les versants pentus à banquettes, de trouver des buissons d'Oleastre bien alignés dans le taillls et vestiges d'oliviers dont les troncs coupés ont été utilisés pour la fabrication d'ustensiles ou pour du bois de chauffage.

La dépopulation de l'espace rural a eu pour conséquence la désorganisation de toutes les pratiques d'utilisation de l'écosystème yeuseraie.

Les chênaies se sont développées en taillis impénétrables et les anciens parcours ont été envahis par des arbustes qui représentent un matériel de choix pour la propagation des incendies. C'est le cas plus spécialement en Languedoc et en Provence où des interventions rapides s'imposent pour dégager de leur sous-bois arbustif ces taillis qui constitueront d'excellents pare-feux naturels.

II est donc plus important, là où en région méditerranéenne on veut utiliser la forêt de Chêne vert pour mettre en place des parcelles de protection ou des aires de loisirs, de convertir le plus rapidement ces taillis plutôt que de s'obstiner à introduire des résineux. Qu'on enrésine là où les potentialités des parcelles permettent un rendement économique et là oủ une forêt doit être reconstituée de toute pièce.

Il s'agit donc de gérer d'une manière différente l'écosystème yeuseraie, de le réorienter afin qu'il réponde aux besoins nouveaux auxquels sont confrontés les hommes tant en ce qui concerne l'utilisation économique, les loisirs, que la lutte contre les feux.

Ainsi, la forêt de Chêne vert aura retrouvé sa place dans une nouvelle organisation planifiée de l'espace forestier naturel. 


\section{BIBLIOGRAPHIE}

ALBERT (A.), JAHANDIEZ (E.). - Catalogue des plantes vasculaires du département du Var. - Paris, P. Klinckosieck éd., 1908.

BARBERO (M.). - Les remontées méditerranéennes sur le versant italien des Alpes. Ecologia mediterranea, $\mathrm{n}^{\circ} 4,1979$, pp. 109-132.

BARBERO (M.), QUEZEL (P.). - Les groupements forestiers de Grèce centroméridionale. Ecologia mediterranea, $\mathrm{n}^{\circ} 2,1976$, pp. 3-86.

BARBERO (M.), QUEZEL (P.). - La végétation forestiére de la Crète. Ecologia mediterranea, $\mathrm{n}^{\circ} 5,1980$, Pp. 175-210.

BARBERO (M.), QUEZEL (P.), RIVAS.MARTINEZ (S.). - A propos de la valeur bioclimatique et dynamique de quelques essences forestières au Maroc. Ecologia mediterranea, $n^{\circ} 5,1980$, pp. 211-249.

BERNARD (J.). - Premiére contribution de l'analyse pollinique de sédiments marins à la connaissance de l'histoire quaternaire de la végétation provençale. - Thèse de spécialité, Université d'Aix-Marseille, 1971.

BERNARD (J.). - Analyse pollinique d'une séquence marine wurmienne provenant de Méditerranée occidentale. Comptes rendus de l'Academie des Sciences, $n^{\circ} 274,1972$, pp. 6-49.

BOLOS (O. de). - El paisaje vegetal barcelonés. - Universidad de Barcelona, 1962, pp. 1-192.

BOLOS (O. de), MOLINIER (R.). - Recherches phytosociologiques dans l'lle de Majorque. Collecteana Botanica, $n^{\circ} 5,1958$, pp. 699-865.

GENTILE (S.). - Remarque sur la chênaie d'Yeuse de l'Apennin méridional et de la Sicile. Vegetatio, $n^{\circ} 17$, 1969, pp. 214-231.

LECOMPTE (M.). - La végétation du Moyen Atlas central. Travaux de I'Institut national de recherche agronomique de Tunisie, vol. $42, \mathrm{n}^{\circ} 5,1969, \mathrm{pp}$. 1-617.

LOISEL (R.). - Séries de végétation propres, en Provence, aux Massifs des Maures et de l'Estérel (ripisylves exclues). Bulletin de la Société botanique de France, $\mathrm{n}^{\circ} 118 \mathrm{a}, 1971$, pp. 203-236.

LOISEL (R.). - La végétation de l'étage méditerranéen dans le sud-est continental français. - Thése de doctorat es-sciences, Université d'Aix-Marseille, 1976.

LOSSAINT (P.), RAPP (M.). - La forèt méditerranéenne de Chênes verts (Quercus ilex L.). - Paris, Masson, 1978, pp. 129-185.

MOLINIER (R.). - Etudes phytosociologiques et écologiques en Provence occidentale. - Thèse de doctorat és-sciences, Marseille, 1934 (Annales du musée d'histoire naturelle de Marseille et S.I.G.M.A., communication 35a, 1935).

MONDINO (G.P.). - Note écologicche sulla stazione relitta di "Quercus ilex" à Chianoc (Valle di SusaPiemonte). Allionia, $\mathrm{n}^{\circ} 12,1966$, pp. 93-101.

OZENDA (P.). - Perspectives nouvelles pour l'étude phytogéographique des Alpes du Sud. Documents pour la carte de végétation des Alpes, vol. IV, 1966, $198 \mathrm{p}$.

OZENDA (P.). - Sur une extension de la notion de zone et d'étage subméditerranéens. C.R. Soc. Biog., 415 , pp. 92-103.

OZENDA (P.). - Sur les étages de végétation dans les montagnes du Bassin méditerranéen. Documents de cartographie écologique, vol. XVI, 1975, pp. 1-32.

PONS (A.). - Contribution palynologique à l'étude de la flore et de la végétation pliocénes de la région rhodanienne. Thése de doctorat ès-sciences, Université de Montpellier, 1964.

PONS (A.). - Les macroflores quaternaires de France. - Ėtudes françaises sur le quaternaire; VIIJe Congrès international INQUA, 1969, pp. 85-93.

PONS (A.), REILLE (M.), TRIAT (H.), COUTEAUX (M.), JALUT (G.), ONER (S.), PLANCHAIS (N.), VERNET (J.L.). Les données historiques et l'étude de la flore méditerranéenne. - In : La flore du Bassin méditerranéen : essai de systématique synthétique, 1974, pp. 305-325 (Colloques internationaux du C.N.R.S., Montpellier).

PONS (A.), VERNET (J.L.). - Une synthese nouvelle de I'histoire du Chêne vert (Quercus ilex L.) Bulletin de la Société botanique de France, $\mathrm{n}^{\circ} 118,1971$, pp. 841-850.

THINON (M.). - Incidence écologique des reboisements du Mont Ventoux (Vaucluse). Aspects floristiques et pédologiques. - Thèse de spécialité, Marseille, 1979.

TRIAT-LAVAL $\left(H_{\text {. }}\right.$. - Contribution pollenanalytique à l'histoire tardi- et post- glaciaire de la végétation de la basse vallée du Rhône. - Thèse de doctorat d'Etat, Marseille, Saint-Jérôme, 1978.

VERNET (J.L.). - Contribution à l'histoire de la vègétation du Sud-Est de la France au quaternaire. Etude de macroflores, de charbons de bois principalement. - Thése de doctorat és-sciences, Montpellier, 1972 ; Paleobiologie continentale, vol. $4, \mathrm{n}^{\circ} 1,1972$, pp. 1-90. 